\title{
Finite Fermion Density on the Lattice
}

\author{
P. HASENFRATZ and F. KARSCH \\ CERN, Geneva, Switzerland
}

\begin{abstract}
:
The naive way of introducing finite fermion density on the lattice leads to quadratic divergences even for free fermions. The problem is discussed and a simple solution is suggested in this paper.
\end{abstract}

The exciting problems of cosmology and heavy ion collisions [1] call for the introduction of finite temperature and particle density in (non-perturbative) QCD studies. The investigation of QCD under extreme conditions has a special flavour. Rather than attempting to explain and quantitatively derive old, well-known experimental data, we deal with a subject unexplored in laboratory experiments, and the results derived from QCD might influence future experiments.

There is a straightforward way of introducing finite temperature in lattice calculations. The continuum prescription for the partition function in terms of a functional integral can be taken over directly on the lattice. The partition function is defined as

$$
Z=\int \mathrm{D} U_{\ell} \mathrm{D} \psi \mathrm{D} \bar{\psi} \exp \left\{-S_{\mathrm{E}}(U, \psi, \bar{\psi})\right\}
$$

on a lattice, which is finite along the fourth (temperature) direction: $N_{\beta} a=1 / T$ is finite. The gauge and fermion fields satisfy periodic and antiperiodic boundary conditions, respectively.

The lattice approach proved to be powerful in determining the critical temperature and other thermodynamical quantities of pure gauge QCD $[2,3]$.

On the other hand, the introduction of finite particle density is hindered by the problems of defining the chemical potential on the lattice in a satisfactory way [4]. The naïve generalization of the continuum prescription leads to quadratic divergences even for free fermions: in the continuum limit $(a \rightarrow 0)$ the energy density $\varepsilon$ is proportional to $(\mu / a)^{2}$ instead of the correct finite result $\varepsilon \sim \mu^{4}$ (for massless fermions), with $\mu$ being the chemical potential.

Let $H$ be the Hamiltonian of the system and $N$ some conserved quantity. In the following, we shall take $N$ to be the fermion number, the generalization is straightforward. The partition function is given by

$$
Z=\mathrm{e}^{-\beta \Omega}=\operatorname{Tr} \mathrm{e}^{-\beta(H-\mu N)} .
$$

Going to functional integral representation, the presence of a non-zero chemical potential implies a new term in the Lagrangian: $\mu$ times the fourth component of the current the space integral of which gives the conserved quantity $N$.

Let us take free fermions in the continuum. The action is 


$$
\int_{0}^{\beta}\left(\frac{1}{2} \bar{\psi}(x) \gamma_{\mu} \stackrel{\leftrightarrow}{\partial}_{\mu} \psi(x)+m \bar{\psi}(x) \psi(x)+\mu \bar{\psi}(x) \gamma_{4} \psi(x)\right) \mathrm{d}^{4} x
$$

with the usual antiperiodic boundary conditions along the fourth direction. The energy density is defined as

$$
\varepsilon=-\left.\frac{1}{V} \frac{\partial}{\partial \beta} \ln Z\right|_{\beta \mu \text { fixed }}
$$

and is given by the following integral when $T \rightarrow 0$ :

$$
\varepsilon=\frac{4}{(2 \pi)^{4}} \int \mathrm{d}^{4} p \frac{\left(p_{4}+\mathrm{i} \mu\right)^{2}}{\left(p_{4}+\mathrm{i} \mu\right)^{2}+p^{2}+m^{2}} .
$$

$\varepsilon$ is a (mass) ${ }^{4}$ quantity. By power counting, the integral is quartically divergent. There is a trivial part, the vacuum energy at $\mu=0$. By subtracting this term we get:

$$
\varepsilon=-\frac{4}{(2 \pi)^{4}} \int \mathrm{d}^{4} p\left[\frac{p^{2}+m^{2}}{\left(p_{4}+\mathrm{i} \mu\right)^{2}+\boldsymbol{p}^{2}+m^{2}}-(\mu=0)\right] .
$$

The integral is finite (not quadratically divergent) as it should. By performing the $p_{4}$ integral first, two poles are encountered in the $p_{4}$ plane both for $\mu \neq 0$ and $\mu=0$ (fig. 1). The difference is zero, except when both poles lie on the lower half plane for $\mu \neq 0$. This gives a $\theta$ function:

$$
\varepsilon=\frac{4}{(2 \pi)^{4}} \pi \int \mathrm{d}^{3} p \theta\left(\mu-\sqrt{\boldsymbol{p}^{2}+m^{2}}\right) \sqrt{\boldsymbol{p}^{2}+m^{2}}
$$

or

$$
\left.\varepsilon\right|_{m:}=\frac{1}{4 \pi^{2}} \mu^{4}
$$

which is the correct result.

The naïve generalization of this prescription would lead to the lattice action

$$
S=\sum_{x}\left\{\frac{1}{2} \sum_{\mu}\left(\bar{\psi}_{x} \gamma_{\mu} \psi_{x+\hat{\mu}}-\bar{\psi}_{x+\hat{\mu}} \gamma_{\mu} \psi_{x}\right)+m a \bar{\psi}_{x} \psi_{x}+\mu a \bar{\psi}_{x} \gamma_{4} \psi_{x}\right\},
$$

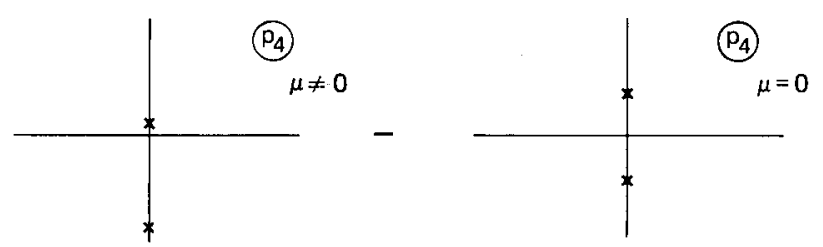

Fig. 1. 
and yields the following result for the energy density $(T \rightarrow 0)$

$$
\varepsilon=a^{-4}\left\{-\frac{1}{4 \pi^{4}} \int_{-\pi}^{\pi} \mathrm{d}^{4} q \frac{\sum_{j=1}^{3} \sin ^{2} q_{j}+(m a)^{2}}{\left(\sin q_{4}-\mathrm{i} \mu a\right)^{2}+\sum_{j=1}^{3} \sin ^{2} q_{j}+(m a)^{2}}\right\}-a^{-4}\{\mu=0\} .
$$

What was a potential problem before, is present here explicitly: $\varepsilon$ is quadratically divergent

$$
\varepsilon \sim(1 / a)^{2} \mu^{2}
$$

The problem is not related to the species doubling implied by eq. (3). The 16-fold degeneracy can be removed by Wilson's prescription, but the problem discussed above, remains. Replacing the current $\bar{\psi}_{x} \gamma_{4} \psi_{x}$ by a point-split form does not help either. In order to obtain a finite result, non-covariant counterterms should be introduced in eq. (3). Although their presence is understandable (there is no Euclidean symmetry for $\mu \neq 0$ ), it would be an awkward way to proceed.

What is the reason that no similar problems occurred in the continuum formulation? The key to understanding is the observation that in the Euclidean formulation of thermodynamics the chemical potential acts like the fourth component of an imaginary, constant vector potential. In continuum QED, for instance, the chemical potential is introduced exactly as a photon field:

$$
e \bar{\psi}_{x} \gamma_{\mu} A_{\mu} \psi_{x}-\mathrm{i} \mu \bar{\psi}_{x} \gamma_{4} \psi_{x}
$$

For this reason an expansion in powers of $\mu$ is equivalent to inserting external, zero momentum photon lines to the amplitude. For instance, the contribution of order $\mu^{2}$ to the thermodynamic potential $\Omega=-(\beta V)^{-1} \ln Z$ is proportional to

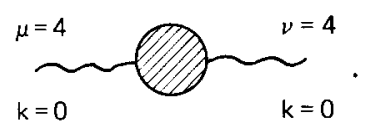

This graph is proportional to the (potentially quadratically divergent) photon mass renormalization which, however, is zero in a gauge invariant formulation at zero temperature (or, due to the plasmon effect, finite at finite temperature). Similarly, the finiteness of contributions of the order $\mu^{l}$ to the amplitude $\Gamma^{(n, m)}$ ( $n$ and $m$ being the number of external photon and electron lines, respectively) follows from the renormalizability of the amplitude $\Gamma^{(n+l, m)}$. Although $l$ factors of the electromagnetic coupling constant $e$ are replaced by the chemical potential $\mu$ and $l$ factors of wave function renormalization are also absent, the missing factors for charge and wave function renormalizations just cancel. This is again the consequence of gauge invariance.*

In eq. (3) this Abelian gauge invariance is violated: $\mu$ does not enter like the fourth component of a gauge field on the lattice. The correct solution is

$$
S=a^{3} \sum_{x}\left\{m a \bar{\psi}_{x} \psi_{x}+\frac{1}{2} \sum_{j=1}^{3}\left(\bar{\psi}_{x} \gamma_{j} \psi_{x+\hat{j}}-\bar{\psi}_{x+\hat{j}} \gamma_{j} \psi_{x}\right)+\frac{1}{2}\left(\mathrm{e}^{\mu a} \bar{\psi}_{x} \gamma_{4} \psi_{x+\hat{4}}-\mathrm{e}^{-\mu a} \psi_{x+\hat{4}} \gamma_{4} \psi_{x}\right)\right\} .
$$
$[5]$.

* The importance of this generalized gauge invariance in the renormalization of theories with finite chemical potentials is emphasized by Baluni 


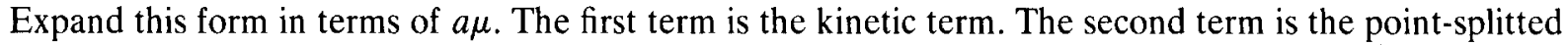
current. However, there are higher order extra contributions-just the appropriate non-covariant counterterms we discussed before. Of course, we shall not expand in $\mu a$-like we do not expand $U_{n \mu}$ in terms of $A_{n \mu}$.

By this prescription, in eq. (9) in the integrand $\left(\sin q_{4}-i \mu\right)^{2}$ is replaced by $\sin ^{2}\left(q_{4}-i \mu\right)$ like in the continuum theory, and performing the $q_{4}$ integration we get

$$
\varepsilon a^{4}=\frac{1}{2 \pi^{3}} \int_{-\pi}^{\pi} \mathrm{d}^{3} q \theta\left(\mathrm{e}^{\mu a}-b-\sqrt{b^{2}+1}\right) \frac{b}{\sqrt{b^{2}+1}}
$$

with

$$
b^{2}=\sum_{j=1}^{3} \sin ^{2} q_{j}
$$

Therefore, we see from eq. (13) that in every corner of the Brillouin zone the $q_{4}$ integration leads in the $a \rightarrow 0$ limit to the expected, correct result for the momentum cut-off $\sim \theta\left(\mu-\sqrt{q^{2}+m^{2}}\right)$, and the resulting energy density is 16 times the usual finite energy density of free fermions at zero temperature.

Using Wilson fermions, the degeneracy is removed and the factor 16 disappears for any $r \neq 0$ as it should $(0<r \leq 1$ is the usual arbitrary parameter in the Wilson action).

Equation (12) can be immediately generalized to the case of QCD. At finite temperature and chemical potential the Wilson action with one flavour has the form

$$
\begin{aligned}
S= & a^{3} \sum_{x}\left\{\bar{\psi}_{x} \psi_{x}-K \sum_{j=1}^{3}\left[\bar{\psi}_{x}\left(r-\gamma_{j}\right) U_{x, j} \psi_{x+\hat{j}}+\bar{\psi}_{x+\hat{j}}\left(r+\gamma_{j}\right) U_{x, j}^{+} \psi_{x}\right]\right. \\
& \left.-K\left[\mathrm{e}^{-\mu a} \bar{\psi}_{x}\left(r-\gamma_{4}\right) U_{x 4} \psi_{x+\hat{4}}+\mathrm{e}^{-\mu a} \bar{\psi}_{x+\hat{4}}\left(r+\gamma_{4}\right) U_{x 4}^{+} \psi_{x}\right]\right\}+\frac{2 N}{g^{2}} \sum_{p}\left(1-\frac{1}{N} \operatorname{Re} \operatorname{Tr} U_{p}\right),
\end{aligned}
$$

where the gauge fields $U$ are periodic, while the fermion fields $\psi, \bar{\psi}$ are antiperiodic along the "temperature" direction. The prescription is very simple: the hopping parameter $K$, related to the quark propagation by one lattice unit along the positive (negative) imaginary time axis is replaced by $\mathrm{e}^{\mu a} K\left(\mathrm{e}^{-\mu a} K\right)$.

Considering the thermodynamic potential at finite temperature, there are quark paths wrapping around the lattice in the imaginary time direction. Only these paths can lead to chemical potential dependence - from ordinary closed paths the $\mu$ dependence cancels. This is understandable: ordinary loops describe virtual pair creation and annihilation, and the chemical potential of quarks and antiquarks is of opposite sign. It follows that it is not advisable to study this system at zero temperature exactly, even if we want to discuss the effect of the finite particle density alone. The hopping parameter expansion (and related iterative methods) breaks down in this case. This fact can also be seen from the explicit result for the energy density of free quarks. At zero temperature $\varepsilon$ is proportional to $\theta(\mu-m(K))$, and this distribution cannot be expanded in terms of the hopping parameter. No similar problem occurs at finite temperature. 


\section{References}

[1] For recent reviews, see:

Q. Shafi, in: Intern. Conf. on High Energy Physics, Lisbon (1981), ed. J. Dias de Deus;

L. Van Hove, in: Quark Matter Formation and Heavy Ion Collisions, Bielefeld (1982), eds. M. Jacob and H. Satz.

[2] L.D. McLerran and B. Svetitsky, Phys. Lett. 98B (1981) 195;

J. Kuti, J. Polónyi and K. Szlachányi, Phys. Lett. 98B (1981) 199;

J. Engels, F. Karsch, I. Montvay and H. Satz, Nucl. Phys. B205 [FS5] (1982) 545; Phys. Lett. 101B (1981) 89.

[3] K. Kajantie, C. Montonen and E. Pietarinen, Z. Phys. C9 (1981) 253;

I. Montvay and E. Pietarinen, Phys. Lett. 115B (1982) 151;

J. Kogut, M. Stone, H.W. Wyld, W.R. Gibbs, J. Shigemitsu, S.H. Shenker and K.D. Sinclair, Phys. Rev. Lett. 50 (1983) 393;

T. Celik, J. Engels and H. Satz, Phys. Lett. 125B (1983) 411.

[4] P. Hasenfratz and F. Karsch, Phys. Lett. 125B (1983) 308.

[5] V. Baluni, Phys. Rev. D17 (1978) 2092. 